\title{
La educación chilena, ¿no se vende? Movilización estudiantil y la configuración del problema público universitario
}

\author{
Juan Pablo Paredes P. \\ Universidad Católica del Maule, Ta lca, Chile \\ Email: jparedes@ucm.cl \\ Camila Araya Guzmán \\ Asistente de Investigación Fondecyt Postdoc tora do № 3170504 \\ Email: carayaguzman@gmail.com
}

Recibido: 02.03.2020 ｜Ａceptado: 06.07.2020

\begin{abstract}
Resumen: El propósito del artíc ulo es describir y comprender el proceso de configuración del problema público de la educación superior chilena, a partir de la movilización estudiantil en defensa de la educación pública, hasta la conformación de una respuesta política institucional limitada la idea de gratuidad (2011-2017). A través de una investigación cualitativa que conjuga diferentes procedimientos de producción de información, y a plicando la perspectiva de la sociología de los problemas públicos, proponemos como hallazgo más significativo la conformación de dos momentos en el proceso, que definen dos a renasy problemáticas diferencia das. El momento de la manifesta ción públic a (2011-2013) asociada a la demanda porel derecho a la educación públic a gratuita, y el momento de la a rena téc nico-instituc ional de la gratuidad (2014-2017). Concluimos reconociendo a lgunos alcances sociopolíticos del proceso, en especial para la movilización estudiantil y su causa pública, a partir de la comparación entre ambos momentos.
\end{abstract}

Palabras claves: Problema público; protestas estudiantiles; arenas públicas; reforma universitaria; derecho a la educación.

\section{Chilean educ ation is not for sale? Sudent mobilization and the configuration of the university public problem}

Abstract The purpose of the article is to describe and understand the process of shaping the
public problem of Chilean higher education, from student mobilization in defense of public
education, to the formation of an institutional policy response limited to the idea of free
education (2011-2017). Through a qualitative research that combines different procedures of
information production, and a pplying the perspective of the sociology of public problems,
we propose as the most significant finding the conformation of two moments in the process,
which define two differentiated a renasand problems. The moment of public manifestation
(2011-2013) associated with the demand for the right to free public education, and the
moment of the technical-institutional arena of free education (2014-2017). We conclude by
recognizing some socio-political scopes of the process, especially for student mobilization
and its public cause, based on the comparison between both moments.
Keywords: Public problem; student protests; public a renas; university reform; education rights 


\section{Educação chilena, não está à venda? Mobilização estudantil e configuração do problema público universitánio}

Resumo: O propósito do artigo é desc revere compreendero processo de configuração do problema público da educação superior chilena, a partir da mobilização estudantil em defesa da educação pública, a té a conformação de uma resposta política institucional limita da à ideia de gratuidade (2011-2017). Atra vés de uma pesquisa qualitativa que conjuga diferentes procedimentos de produção de informação, e aplicando a perspectiva da sociologia dos problemas públicos, propomos como achado mais significativo a conformação de dois momentos no processo, que definem duas arenas e problemáticas diferenciadas. O momento da manifestação pública (2011-2013) associada à demanda pelo direito à educação pública gratuita, e o momento da a rena téc nico-institucional da gratuida de (2014-2017). Conc luímosrec onhecendo a lgum esc opo soc io polític o do proc esso, princ ipa Imente para a mobiliza ção estudantil e sua causa públic a, a partir da compa ração entre os dois momentos.

Palavrasc have: Problema públic o; protestosestuda ntis; a renas públic as; reforma universitá ria; direitos educacionais.

\section{Como citar este artículo:}

Paredes P, JP y Araya Guzmán, C. (2020). La educa ción c hilena, ¿no se vende?

Movilización estudiantil y la configura ción del problema público universitario. Polis Revista La tinoa merica na, (57), 251-271. doi: http://dx.doi.org/10.32735/S0718-6568/2020-N57-1573

\section{Introducción}

«(...) Este proyecto no sólo posterga la gratuidad probablemente al año 2070, sino que peor aún, consolida el CAE hasta ese periodo»(en Marín \& Ra mírez, 18 de julio 2017), seña ló el diputado G. Jackson, frente a la aprobación del proyecto de Reforma a la Educación Superior en la Cámara de Diputados. El parlamenta rio frentea mplista calificó de insuficiente el proyecto del segundo Gobiemo de M. Bachelet, porno elimina r el c réd ito con aval del Estado (CAE) ni la bancarización de la educación superior. Contrariamente, mantiene su dependencia del cumplimiento de metas fiscales o del subsidio a la demanda educativa sin potenciarla educación pública, subsumida en una indefinición de su significado.

El proyecto, fue respuesta a la movilización estudiantil que impactó a la opinión pública nacional, desde el2011, con su demanda de la educa ción como un derec ho social, logrando situar la crisis de la educación pública como un problema de connotación pública. ¿Cómo fue el proceso de problematización y crítica del estatuto mercantil de la educación? ¿Cuáles fueron las definiciones en competencia? ¿Cuáles fueron los alcances instituc ionales y cultura les para el modelo educativo? ¿Qué implicó para la política y para la movilización estudiantil el proceso de problematización? Son algunas de las preguntas que alientan el trabajo, y que intentamos abordar desde la perspectiva de los problemas públic os.

Proponemos como tesis que el conflic to pasa de un escenario antagónic o (la arena callejera ) -con su lógic a, rela ciones, lenguajes y prácticas- entre el mundo estudia ntil moviliza do 
y la institucionalidad política (gobiemo y Estado), tensionando la forma tradicional de procesar los conflictos desde el retorno a la democracia, a una arena de controversia tec nocrática-institucional, con otra lógica, relaciones, lenguajes y prácticas, entre actores diversos y más diferenciados, tanto sociales y políticos. Tal institucionaliza ción significa reencauzar el conflic to hacia los procedimientos habituales para procesarlos, a unque ello no necesariamente significó restituir sin perjuicios la forma política que ha primado en el país desde la década del 90'.

El texto se estructura de la siguiente manera. Primero, introducimos la perspectiva de los problemas públic os para estudiar el conflic to estudiantil reciente en Chile y exponemos los alcancesmetod ológic os. En segund o lugar, contextua liza mosel proc eso de merc antiliza ción de la educación en un marco de más de treinta años, así como las respuestas desde la moviliza ción estudia ntil pre 2011. Tercero, proponemos una interpreta ción del conflic to estudiantil a partir de la periodización del proceso desde la óptic a de losproblemaspúblicos. Finalmente, a manera de conclusión, realizamos una reflexión sobre la pertinencia de la a proxima ción desde los problemas públic os para interpreta rel conflic to estudia ntil rec iente y sus a lcances sociopolític os mayores, para la configuración de la democracia en Chile.

\section{En tomo al estudio de los problemas públicos}

Desde la sociología de losproblemassociales, estosse entienden como definicionesde una situa ción rea lizada por actores sociales, mediante una serie de procesos de signific ación e imputa ción de sentido (Schilla gi, 2011). La propuesta Constructing social problems (Spector \& Kitsuse, 1977), presentó una posición constructivista para estudiar problemas sociales, debido a la incapacidad de las ciencias sociales de dar con una definición objetiva de ellos, proponiéndose como altemativa al positivismo y al funcionalismo a la hora de estudiar situa cionesy procesos de conflic to sociopolítico. El supuesto basal dice que la realidad social resulta de un proceso de construcción subjetiva de los actores sociales, sin posibilidades de reducirla a sus condic iones objetiva s o na tura les (Spector \& Kitsuse, 1977).

La perspectiva de losproblemaspúblic osse fomula alineada a la propuesta construc tivista, desde la obra de J. Gusfield (1981), a unque recientemente, se ha revita liza do por la emergencia de una serie de trabajos franceses llamados «pragmatistas», distanciándose del construc cionismo (C efaï, 2014). El estudio de los problemas públicos, en una clave próxima al pragma tismo (Cefaï, 2014; 2011; Trom, 2008), a cepta el principio de definición del problema por losactores (Gusfield, 1981; Spector \& Kitsuse, 1977), pues comparten el supuesto que los a c tores so cia les son competentes en sus mod os de a c tua c ión y justific a ción de las situacionesen que partic ipan. Es dec ir, sa ben lo que hacen y pueden justific a rlo (Na rda cc hioni \& Acevedo, 2013).

Sin embargo, se distancia de aquel, porque un problema públic o requiere de la atención y responsabilización de una autoridad polític a e institucional, de preferencia el Estado, para configurarse como tal. Complementa lo anterior, la suposición que la definición de una 
situa ción problemátic a a lcanzará el estatus de problema público al relaciona ro con gramáticas cultura les que los legitiman (Cefaï, 2014; Nardacchioni \& Ac evedo, 2013), dotándolos de un carácter moral a socia dos a criterios de injusticia (Trom, 2008). Entonces, partir de la movilización de un actor colectivo que define un problema, mediante formas de categorización, y lo hará perceptible socialmente vía un proceso de publicitación, que tendrá connota ciones mora les o de justicia, concitando la atención de la autoridad política y su responsa biliza ción en la superac ión del problema. Ta les son las característic as de un problema público y su diferencia con un problema social.

Pa ra D. Cefaï (2014; 2011), estudiar conflic tos desde los problemas públic os, permite da res inteligibilidad mediante operaciones de descripción e interpretación en base a las claves a na lític as de atribución de responsabilidad y propiedad del problema, la categorización y public itación del mismo y la conforma ción de a renas de controversias públicas.

La propiedad del problema (Gusfield, 1981), refiere a la capacidad de ciertos colectivos e instituciones de constituirse en las «voces autorizadas» del problema, inscribiéndolo en la vida social, al definir sus causas, los causa ntes y las soluc iones posibles, lo que entreteje un conjunto de relaciones en tomo al problema. Entre ellas, la responsabilidad en el origen y la resolución del problema. Gusfield (1981), diferencia la responsabilidad causal, vinculada al origen del problema, de la responsabilidad política, vinculada a la obligación moral y legal de solucionarlo. Por ende, la responsabilidad política se vuelve relevante en el horizonte que el problema puede ser superado. Sin embargo, la propiedad, la causalidad y la obligación, no necesariamente deben coincidir en un mismo actor, lo que dinamiza la disputa pública.

La categorización implic a definir el problema a través de ciertasconceptos y no otros, que intenta n espec ific ar sus dimensiones y a lc ances (C efaï, 2014), mientras que la public ita ción remite a las a ctividades y categorías desplegadas por los a c tores implic ados en el problema, que mediante sus performances, voc abularios, reclamos, busc an lograr la adhesión de públic osy la moviliza ción emotiva de audiencias (Nardacchioni \& Acevedo, 2013). A tra vés de ella, se exponen públic a mente modalidades diversas de definir, juzgar, categorizar, percibir y sentir. La public ita ción es una operación compleja destina da a la configura ción y la emergencia de públicos, ya sean colectivos a ctivos o audiencias receptivas a la moviliza ción y sus demandas (Cefaï, 2014).

Las a renas de controversias, remiten a espa cios de disp utas y controversia s del que partic ipan a c to resque despliega n opera c iones de c lasific a c ión, dra matiza ción, retóric a y de fuerza (Cefaï, 2014), mediante las cuales se define el carácter público del problema. Hilgartner \& Bosk (1988) propusieron las arenas de controversias para referir al proceso de definición, selección y priorización de problemas públicos. Según los autores, estas arenas tienen su propia lógica de funcionamiento, que pemiten encuadrar los problemas bajo ciertas definiciones. Una arena es tanto una escena de realizaciones (performances) y un espacio de lucha (combate) en los que son configura dos losproblemaspúblicos, con sus propias reglas 
de juego (lenguajes, proc edimientos, nitua les, persona jes a credita dos, está nda res de expenencia) que se inscriben en una «gramática de la vida pública »que demarca lo aceptable y legítimo en ella (Lemiux, 2017; Nardacchioni \& Acevedo, 2013; Cefaï, 2011).

La public itación del problema a dquirirá su legitimación al vinc ula rla a ciertas reglasy ca tegońa s socia Imente vá lidas, denomina das gramátic as públicas (Na rda cc hioni \& Acevedo, 2013). Para que lo anterior se produzca, en síntesis, es necesario que la categorización y public itación del problema sea capaz de configura r públic osque reconozc an en y se identifiquen con, el problema (Cefaï, 2014, 2011; Na rda cc hioni \& Acevedo, 2013). Por último, las dinámic as conflic tivas se dan en determina dos escena rios públic os en las que se desa rrolla la disputa en una relación tríadica-denunciantes o agentes, antagonistas y públicos (Na rda cc hioni \& Márquez, 2016; Cefaï, 2014; Na rdac chioni \& Ac evedo, 2013).

\section{Alcances Metodológicos}

Empíric a mente, los problema s públic os permiten modalida des de descripción e interpretación de situa c iones conflic tivas, a partir de las actua ciones y disc ursos de los ac tores implicados, como de los públic os afecta dos por ella (Lemiux, 2017; Cefaï, 2011). Pa ra rea lizar tal descripción comprensiva, se observan las formas de dramatización (performances), de categorización y de narración en la trayectoria de un problema público y su relación con gramátic as de la vida públic a (Cefaï, 2011; Nardac chioni \& Márquez, 2016; Nardac chioni \& Acevedo, 2013; Paredes, 2013). En tal sentido, utilizamos una perspectiva cua litativa, detallada a continuación, para darcuenta del proceso de configuración del problema público universita rio.

En tomo al conflic to por la educación superior (2011-2017), realizamos la producción de informa ción en dos etapas. Primeramente, a partir de una investiga ción doctoral, se siguió a la movilización estudiantil en sus manifesta ciones públic as y dinámicas orga niza ciona les en Santiago, mediante la observación participante de ellas, la conversación situacional con activistas, un registro fotográfico de las protestas, el uso documentos de las organizacionesy materia les de prensa. Se participó de un total de 28 marchas estudiantiles en Santiago, entre los años 2011-2013. El segundo momento, en base a una investigación posdoctoral, se rea lizó a partir de entrevistas a activistas y militantes de alguna fuerza política o colectivo estudiantil universitario y que participaran del proceso de movilización. Se complementó con el uso de material de prensa digital en tomo al conflic to estudiantil. Se entrevistó a un total de 40 a c tivista sy militantes del norte, centro y sur de Chile, durante dos años, y elaboramos un archivo noticioso desde el 2014 al 2017, sobre el conflic to estudiantil por la educación pública y la reforma que le siguió.

El tra bajo a na lític o se inspira en las categońas y principios metodológic os de los problemas públic os, en particular la hipótesis de continuidad y el principio de seguimiento a los actores. La primera refiere a relación de continuidad entre todas las dimensiones implicadasen 
el proceso, como lo singulary lo general o lo colectivo y lo instituc ional. Para nuestro caso, la del espacio de la movilización y la esfera política, o la calle y las instituciones. Por su parte, seguir a los actores implica describir y comprender las formas en que los actores entran en relación entre ellos, definen situaciones, responden unos a otros, sin presuponer ningún telos al proceso. Es una perspectiva que ve a la acción «haciéndose»en el proc eso mismo de su realización, privilegiando la dimensión dinámica de la vida colectiva (Nardacchioni \& Acevedo, 2013). En nuestro caso, inic ia con las manifestaciones públic as del mundo estudiantil en mayo del 2011, para luego seguir su dinámica hacia la institucionalidad. Ambos principios validan la operación de periodizar como una forma de dar cuenta desc niptiva e interpretativamente del proceso de cuestiona miento del modelo neoliberal de educación supenior, a partir del momento de la denuncia pública de un actor colectivo.

La periodización elaborada, se funda en los conceptos de causa pública, movilización y performance estudiantil, c a tegoriza ción y publicita ción, propiedad del problema y arenas de controversias. Sostenemosque la red a rtic ula da entre talesconceptos, pemite darcuenta de diferentes momentosen los que se formuló diferenciadamente el problema público de la educa ción universita ria en Chile. Con ello, pretendemos mostrar las formas, rela ciones y alcances que tuvo el proceso de movilización estudiantil por más de cinco años. Cada momento propone la cristalización de lógicas, lenguajes, actuaciones, relaciones y espacios que permiten captar ciertas configura ciones problemátic as que producen efectos en lo social y lo político.

Antes de proponer la periodiza ción, es nec esa rio contextua liza r el conflic to de la educación pública chilena.

\section{E modelo mercantil de educación y las c ńticas estudiantiles pre 2011}

La dictadura militar chilena implementó una serie de reformas estructurales en el modelo de sociedad, hacia uno neoliberal (Moulian, 1997; Lechner, 2003; Rojas, 2012). Reformasque termina rán legitimando su transformación, bajo la idea de su modemización, caracterizado por un rol subsidiario del Esta do frente al merca do, la privatiza ción de bienesy servicios, o la reducción del gasto público. Para Garretón y Espinosa (1992), lo que cambió es la relación entre política, mercado y sociedad civil, hegemonizada por la lógica del mercado. Lógica que se mantuvo, con ciertos cambios, durante la transición democrática (Madariaga, 2019; Atria, 2014).

La modemización neoliberal en educación significó pasar de un sistema centraliza do con preeminencia estatal y su lógic a, a uno mixto dominado por el mercado, la competencia y una creciente partic ipa ción del sector priva do. El Esta do a sumió un rol subsidia rio, regulador y de intervención focalizada en cierta población en condiciones de desigualdad (Slachevsky, 2015; Alarcón-Leiva et.al., 2014), constituyendo la «educación de mercado». Bajo elargumento de mejorar la eficiencia del Esta do, en 1981, se formuló la Ley General de Universida des (LGU), que definió el rol subsidia rio del Esta do y la creación de pla nteles priva- 
dos, mediante la modalidad del autofinanciamiento, fomentando las ideas de libertad de enseñanza (lic eosy universida des) y de elec ción (estud ia ntesy susfa milias). Lo a nterior, bajo la premisa de mejora paulatina en la calidad de la educación y la eficiencia en el manejo de recursos, según la lógica de la demanda y la oferta (Assael, et.al., 2011).

La herencia dictatorial en el modelo universitario continuó en democracia ${ }^{1}$. Se consolidó la idea de la educa ción como meca nismo de a scenso social, a hora neolibera liza do, porende, la ma trícula universita ria se multiplicó y nuevas universidades na cieron para responder a la creciente demanda por educación superior. Coherentemente, el año 2005 se implementó el Crédito con Aval del Estado (CAE) para mejorar las tasas de acceso a la universidad, aunque terminó generando resultados perversos, debido al masivo endeudamiento estudiantil y de sus familias (Krememan \& Páez, 2016).

El modelo neoliberal de educación superior se fomentó estratégic amente, para afrontar el desafío de expandir la matríc ula universitaria, al a umentar la inversión en ca pital huma no, extend iendo el merca do educ a cional al sectorfina nciero y de negocios, mediante el ingreso de créditosy la banca (Assael, et al., 2011; Krememan \& Páez, 2016). Cultura Imente, los treinta años de neoliberalización en educación, reforzaron la ideología meritocrática y el esfuerzo ind ividual como pila res so cia les (Ara ujo \& Martucc elli, 2012). En tal lógic a, el modelo educacional de mercado promueve una modalidad de vida social sustentada en la idea de capital huma no y la definic ión de la educa ción como un bien de consumo (Ramírez, 2017). Imponiéndose como modo de vida, un paradigma del mercado por sobre uno de lo públic o (Atria, 2014).

Desde los problemas públicos, un modelo institucional se acompaña de una gramática cultural y pública que sostiene su funcionamiento. Como guía y orientación, en tanto conjunto de reglas y categońas que habilitan realizar determinadas actuaciones y juicios (Lemieux, 2017). Tales reglas, operan como un saber hacer y decir implíc ito, como aquello dado por senta do (Garfinkel, 2006), pero no por ello son estruc tura ina movible. Son reglasy ca teg oriza ciones diná mic as, a c tua liza da sen la acc ión so cial y con potencial de redefinición del sentido sociocultural. Por ende, las gramáticas públic as son ta nto de a c ción y sentidos (Lemiux, 2017),

La lógic a mercantil, sus reglas y categonizaciones, desbordó lo educacional para filtrarse a la Sociedad (Ruiz, 2015; Rojas, 2012), materializá ndose políticamente como una democracia de losacuerdos, del funcionamiento de lasinstitucionesy de la erradic ación del conflicto. Con una ciudadanía despolitizada, atomizada y conducida por funda mentos económic os (Moulián, 1997), produc iéndose un défic it ciudadano ajustado al modelo social impuesto en dic tadura (Paredes, 2011; Báez, 2017). Bajo la idea de gobemabilidad se dota al proceso de estabilidad política y se define el lugar peniférico de la ciudadanía (Paredes,

\footnotetext{
${ }^{1}$ Asegurado con la aprobación en el ocaso de la dictadura de la Ley Orgánica Constitucional de Enseñanza (LOCE).
} 
2019). Donde el consumismo y la innovación, pasaron ser ejes centrales de la construc ción identitaria del país y de búsqueda del reconocimiento individual (Moulian, 1997; Araujo \& Martuccelli, 2012).

El modelo neoliberal y la gramática de la gobemabilidad, favorecieron la consolidación de una democracia de baja intensidad (Rovira, 2007). Este peńodo estuvo marcado por el dictum de «la medida de lo posible»: justicia, igualdad, reconocimiento, derechos, en un nivel controlado con tal de no fomentar el desorden, ni invitar al conflic to social. ${ }^{2}$ Orden, consenso y seguridad, fueron laspala bras clave. «as instituc iones func ionan» fue el corolario ${ }^{3}$, que limitó el conflic to social y los temas políticamente polémicos, a los fomatos y márgenes instituc iona les definidos por la constitución de 1980. Las instituciones procesan y resuelven los conflictos, mediante sus actores (polític os y expertos), sus procedimientos y lenguajes, sin incluir la óptic a ciudadana. El retomo a la democracia consistió, entonces, en una limita ción de la actuación polític a ciudadana, sustituida por su inclusión en el mercado, basada en una gramátic a de la gobemabilidad que artic uló exitosa mente orden y consenso de la instituc ionalidad con ciertas formas culturales (Paredes, 2019; 2018).

No obstante, los estudia ntes c hilenos se moviliza ron contra tal modelo. Aunque, inic ia lmente la transición a la democracia se caracterizó por la desarticulación de las federaciones estudiantiles debido a los efectos de la despolitiza ción anterior (Muñoz \& Durán, 2019). Posteriomente, las federaciones se reactivan en base a ciertas dinámicas organizacionales, promovidas por las J uventudes Comunistas (J J.CC) o colectivos polític os de izquierda, por ejemplo «a Surda», como expresión de la confluencia de grupos vinculados a la lucha contra la dictadura (Thielemann, 2017; Braghetto, 2013).

Lo anterior, significó una recomposición del campo sociopolítico universitario, que extiende la luc ha anti-dic tadura a la herencia del modelo (Muñoz\& Durán, 2019). Ejemplo de este periodo son las movilizaciones a fines de los 90“por el arancel diferencia do o el «moc hilazo» del año 2001, por el uso y extensión del pasaje escolar, a mbas como antecedentes de las futuras moviliza ciones estudia ntiles (Donoso, 2014).

El 2006 se produce la emergencia estudiantil más signific ativa previa al 2010 (Donoso, 2014; Aguilera, 2011), con el lla mado movimiento pingüino ${ }^{4}$, al punto que para algunos autores fue un punto de inflexión la capacidad estudiantil de organiza ción y protesta, como en su incidencia en la agenda pública y política (Muñoz \& Durán, 2019; Vera, 2011).

Los pingüinos posicionaron en la esfera pública la crítica al modelo educativo y su

\footnotetext{
${ }^{2}$ La oración del fallecido Presidente Aylwin fue: «La conciencia moral de Chile exige que se esclarezca la verdad y que se haga justicia, en la medida de lo posible.» Publicada en El Mercurio, 13 de junio de 1990.

${ }^{3}$ Ex-Presidente Ricardo Lagos, señaló que «En Chile las instituciones funcionan» en el marco de la solicitud de justicia por la violación a los DDHH durante la detención de Pinochet en Londres para afimar que en el país las instituciones democráticas se están consolidadas.

${ }^{4}$ Debido al uniforme utilizado en los colegios y liceos que se asemeja a la figura de los pingüinos.
} 
cuestionamiento (Donoso, 2014; Aguilera, 2011; Vera, 2011). La fuerza inusita da del movimiento pingüino, llegó a proponer como objetivo central la derogación de la LOCE. Tal demanda planteó la nec esidad de cambiarla ley e implementar una nueva con un rol más a ctivo del Esta do, proponiendo la «desmunic ipa lizac ión»y denuncia ndo el luc ro en la educación (Pic azo \& Pierre, 2016; Donoso, 2014; Vera, 2011).

La movilización «pingüina», politizó el conflicto por la educación pública, mediante el cuestiona miento a las polític as educ a tiva s de ca rá cter neoliberal. El primergobiemo de M. Bachelet (2006-2009), respondió a las demandasestudiantiles, mediante un Consejo Asesor Presidencial para la calidad de la educa ción, conformado por representantes del mundo polític o, técnic y y social; destina do a guiar la discusión en tomo a la Ley General de Educación (LGE), como reforma de la educa ción pública (Vera, 2011). Su funciona miento culmina en la margina liza ción de las vocessocia les, ciuda da nasy estudia ntilesen su formula ción, al punto que los representantes estudiantiles abandonan la instancia (Picazo \& Pierre, 2016), reafimando la gramática de la gobemabilidad y su lógica institucional.

Aquella movilización dividió a guas en el debate público y político, separando a los sectoresen favor de mantenero refo ma rsuperfic ia Imente el modelo, de las posic iones estudiantiles que buscaban el fin de la merc antiliza ción. El 2008, va rias universidades y colegios se moviliza ron en contra de la promulga ción de la LGE que fue diseñada por la Concertación y la Alianza en respuesta a la «revolución pingüina», y que sostenía algunosprincipiosestruc tura les de la LOCE (Vera, 2011).

El periodo post 2006, se logran identific ar indicios y huellas de unidad entre secundarios y universita rios fomenta ndo una infra estructura organiza c ional y de causa spública sque será relevante al futuro (Paredes, 2019; Donoso, 2014). Sin embargo, a pesardel rechazo de los estudiantes, el año 2009 se promulga la LGE, una derrota para el segmento estudiantil secundario, generando un proceso de repliegue del conflic to educacional. En paralelo, durante el perío do 2005-2010 se evidencia un aumento del endeudamiento significa tivo de los estudiantes de educa ción superior debido al uso de créditosc on a ltos intereses, a dministradosporla banca privada y con el CAE. De esta manera, los pilares de educación pública, gratuidad, mayor presencia estatal y la denuncia del aumento del endeudamiento, fueron construyendo el camino para la configuración de un problema público que marcó la vida polític a chilena poral menos cinco años.

\section{Del problema público de la educación a la arena de la gratuidad}

Desde los problemas públicos, proponemos periodizar el ciclo de movilización estudia ntil post 2010, en dos momentos. El primero, remite a la definición del problema público de la educ a ción superior desde la moviliza ción estudia ntil, que va desde el 2011 al 2013. El segundo, refiere a la conformación de una arena de controversia institucional en tomo a la reforma de la educación pública en clave de gratuidad. A continuación, realizamos un 
a nálisis descriptivo-comprensivo de cada uno de los periodos, para después señalar sus (dis)c ontinuida des.

\section{Primer momento: la calle como arena de disputa política}

El 2011 se caracterizó por la masividad la lucha callejera. Las manifestaciones durante el a ño esc ala ron en ma sivida y y ra dic a lización, en una ola progresiva de acciones de protesta sin mayores precedentes en el marco transicional (Segovia \& Gamboa, 2012). Variadas fueron las formas de manifestación públic a que definieron el repertorio de la movilización ese año, marcado por el carác ter lúdico y heterogéneo de la contienda estudiantil (Paredes, 2019; 2018). Aunque, el recurso característic o del repertorio de protesta del perio do fue la marcha (Cuevasy Paredes, 2018).

Bajo una coordinación nacional, a través de la Confederación de Estudiantes de Chile (CONFECH) y con la partic ipa ción de organiza ciones de estudia ntes sec undarios, como la ACES (Asamblea Coordinadora de Estudiantes Secundarios) y la CONES (Coordinadora Nacional de Estudiantes Secundarios), la organización funcionó en asambleas periódicas para tomar decisiones siempre con el respaldo de las bases, como lo señalan ex dingentes universita rios del periodo (J a ckson, 2013; Figueroa, 2013).

Las marc has se tomaron las calles de las ciudades más importantes de Chile. La denuncia de la mercantilización de la educación superior y su reducción a un bien de mercado, expresadosen la máxima «fin al lucro», rápidamente resona ron en el espa c io público, junto a otras categoría como endeudamiento, cliente, bancarización, becas, subsidios, abandono de lo público (Paredes, 2019) Todas ellas puestas en relación dura nte la serie de manifestac iones públicas masiva sque concitaba n el a poyo transversal de la ciudadanía, a partir de mayo y durante casi todo el año.

Los estud ia ntes moviliza dos deja ron de ser simples estud ia ntes c on sus deriva dos merc a ntiles, «clientes», «usuarios», «deudores», para pasar a ser «estudiantes en la lucha». En la calle, denunciaban el daño que la mercantiliza ción les produjo tanto a ellos como a sus familias. Actuando de manera colectiva en un proceso de agitación social modificaron su identidad colectiva, fomentado la formación de una ciudadanía de tipo activista (Isin, 2009).

Dura nte ta les jo madas, estudia ntes y ciuda da nos moviliza d os delimita ron los conto mos del problema educativo, siendo los dueños de su definic ión públic a (Gusfield, 1981). Definieron su contenido crítico en el lucro a sociado al negocio de la educación y el abandono de lo públic o, junto con una propuesta de superación en base a un horizonte a firma tivo, «educación pública gratuita» basado en el derecho a la educación. Acusaron que la instituc ionalidad polític a y los gobiemos democráticos, mantuvieron la injusticia social derivada de la dictadura, refinándola mediante la incorporación del mercado y a la banca en la educ a ción superior. Definiénd olosc omo los responsa blesde la situa ción y definiénd ose a sí mismos, y sus fa milias, como las víc timas de la injusticia (Pa redes y Otárola, 2019). 
Por otro lado, las performances estudiantiles adquirieron fuerza icónica y una potencia moral (Alexander, 2010), al captar la atención de la ciudadanía. El despliegue de dramatizaciones y puestas en esc ena con un fuerte componente estético dieron a las marchas un ca rác ter lúdic o y festivo, combiná nd olas con expresiones de carácter más violento (Pa redes, Ortiz y Araya, 2018), a dquiriendo una suerte de aura de sentido (Alexander, 2010), que conectó con la ciuda da nía. Media nte lasperformancesde protesta, la ciudadanía se identificó con la movilización y su causa, al mismo tiempo que reforzó con su apoyo intemamente la identidad colectiva de la movilización (Trom, 2008; Favre, 1990). La alegría y el orgullo que las manifesta ciones genera ron, definieron el marco emocional e identita rio del periodo (Paredes y Otárola, 2019). La estrategia de publicita ción de la educación pública gratuita y el fin al lucro, se fundó en un proceso activo de manifestaciones públicas que logró hacer sentido en la ciudadanía, como lo expone una de las entrevistadas.

«El 2011 fue un año de ruptura, yo creo. En varios sentidos, como concebíamos nosotros la moviliza ción social, política, estudia ntil, porque efectiva mente genero un cambio en el sentido común, que yo creo es como, el cliché del año $2011 . .$. ehhh...y de modo particular... claro, nosotros muy al sur de chile, como siempre súper al margen que pasaba siempre, socialmente... nos vimos involucrados de una manera súper protagónica...» (Estudiante universitaria, universidad pública, zona sur)

El aura de las protestas estudiantiles y su conexión con la ciudadanía, sorprendieron a la opinión públic a y limita ron la respuesta de la política. Por ejemplo, el «G ran Acuerdo Nacional por la Educación»(GANE) ${ }^{5}$, propuesto por el gobiemo, no tuvo eco alguno en la movilizac ión, ni en la disc usión política, ni en la ciudadanía. Entonces, la estrategia del gobiemo se redujo a la represión policial de las manifestaciones estudiantiles. El 04 de agosto de ese año se mantiene en la memoria de la ciudadanía chilena por ser la jomada de protesta estudiantil más violenta, debido a la alta represión policial, fecha que se conmemoró posteriomente como el día de la dignidad estudiantil (Moreno, 4 de agosto 2014).

En el periodo 2012-2013 baja la intensidad de la moviliza ción estudia ntil, dejá ndose ver con altibajos. Particulamente, el 2012 que se produce un momento de ajuste de los liderazgos al interior de la CONFECH y a sumen otros actores del mundo educ a cional las banderas de la lucha (bloque de conducción), como los secundarios y los profesores, pero sosteniendo una continuidad de las demandas. El 2013 la lucha callejera estudiantil se retomó como forma de presión al mundo político, debido a las elecciones presidenciales de Octubre de ese año, restituyendo la demanda por la educación pública gratuita y de calidad en el espac io público. Lascausas públic as del fin al lucro y formula ción de la educación pública gratuita en su conjugación restituyeron el vocabula rio de los derec hos socia les en el espacio público. Apelar a los derechossociales, a la educación como derecho y al valor de lo público, se volvió una lengua común y cotidiana. La c ńtic a contra lo privado que se transa en el merc ado y la reducc ión de la educa ción a bien de consumo, ta mbién podrá enunciarse

\footnotetext{
${ }^{5}$ Medidas del GANE: Fondo para la educa ción de 4mil millones de dólares; reducción de la tasa de interés del Crédito con Aval del Estado; aumento de becas para la educación técnico-profesional; creación de una nueva institucionalidad; entre otras.
} 
públic a mente sin esperar censura o sentir vergüenza. Constituyendo un marco emocionalmoral de dignidad ciudadana, que dotó al momento 2011-2013 de un carácter discursivo eminentemente moral (Cefaï, 2014; J asper, 1997), por sobre lo técnico y lo instrumental.

En esos años, el ojo público se volcó sobre la movilización estudiantil, sus formas de actuación y demandas. Los actores estudiantiles generaron un efecto de identidad pública cohesionada, que mantuvo respetabilidad y legitimidad (Tilly, 2008) en la ciudadanía. Fueron ellos quienes se adueñaron del problema público de la educación, definiendo sus contomos, contenidos y horizontes. Public itaron su causa mediante un conjunto de actuaciones públicas expresadas como marchas que combinaban formatos tradicionales de contesta ción, liga dosa momentos de enfrenta miento, con otros momentos másperformátic os, con el fin de fomentar un estado de ánimo y transmitir un mensaje con alcance moral (Trom, 2008; Paredes y Otárola, 2019). Ello se tradujo en un altísimo respaldo público a la demanda estudiantil ${ }^{6}$ y un fuerte compromiso con la movilización estudiantil, aunque en descenso los años venideros. Como lo expone una entrevistada:

«un período de alta esperanza, esperanza y contenido (...) ya después como 2014 comienza, a decaer (...pero...) no tiene que ver con el año, tiene que ver con las reformaso la imposibilidad de lograrcambios a través de presión social» (Estudiante universitaria, universidad privada, zona centro)

Aunque a fines del 2013, la demanda estudiantil generó efectos en la agenda política. Ingresó a la polític a de la mano de las elecciones presidenciales, al estarincomora da en la mayońa de los programas de gobiemo, especia Imente en las candidaturas progresistas y de izquierdas, entre ellas la de M. Bachelet. La únic a candidatura opositora a la educación pública gratuita fue E. Matthei de la Alianza por Chile. Como se expresa en el siguiente cuadro 1:

\section{Cuadro 1}

Candidatos/as Presidenciales

\begin{tabular}{|c|c|c|c|}
\hline Candidato/a & Posición Política & $\begin{array}{c}\text { Educación Pública } \\
\text { Gratuita }\end{array}$ & Fin al lucro \\
\hline Roxana Miranda & Izquierda & Si & Sí \\
\hline Marcel Claude & Izquierda & Sí & Sí \\
\hline Marco Enríquez-Ominami & Centro Izquierda & Sí & Sí \\
\hline Michelle Bachelet & Centro Izquierda & Sí & Sí \\
\hline Alfredo Sfeir & Centro & $\begin{array}{l}\text { Relación sector } \\
\text { público-privado }\end{array}$ & No refiere \\
\hline Ricardo Israel & Centro & $\begin{array}{l}\text { Relación sector } \\
\text { público-privado }\end{array}$ & Sí \\
\hline Franco Parisi & Derecha económica & $\begin{array}{l}\text { Relación sector } \\
\text { público-privado }\end{array}$ & No refiere \\
\hline Tomás Jocelyn-Holt & Derecha Tradicional & $\begin{array}{l}\text { Educación Gratuita } \\
\text { focalizada }\end{array}$ & No refiere \\
\hline Evelyn Matthei & Derecha Tradicional & No & No \\
\hline
\end{tabular}

Fuente: Elaboración propia en base a las propuestas programáticas de los/as candidatos/as.

\footnotetext{
${ }^{6}$ Según Adimark en noviembre del 2011 más del 70\% de los encuestados apoyaba la demanda estudiantil, a unque cerca del $50 \%$ de ellos cuestionaba los modos de presentar la demanda, comenzando los cuestionamientos a la forma protesta. En septiembre del 2012 la aprobación se mantuvo en el 70\%, pero aumentó la desaprobación a la protesta, alcanzando sobre el 60\%. En noviembre del 2013, debido al contexto de elecciones, la aprobación de las protestas alcanza solo al 23\% de los encuestados.
} 
Esto configuró otro escenario para la demanda de la educación pública y para el movimiento estudiantil, a partir del 2014, aunque contaría con representación política en el parlamento. De esta forma, la movilización estudiantil lograba ciertos efectos en el campo político (Montero, 2019), que abren paso a la discusión sobre la reforma de la educación pública.

\section{La arena político-institucional de la gratuidad como forma de contener el conflicto}

La segunda presidencia de M. Ba chelet (2014-2017), incorporó en su programa de gobiemo la causa de la educación pública, conformando una triada de promesas democráticas con las que fue electa. Reforma a la educación, reforma tributa ria y reforma constitucional, fueron las clavesciudadanas con las que el gobiemo propuso desmarcarse del legado de la dictadura. Lo que dio pie a la conformación de una a rena institucional en tomo al problema de la educación superior.

No obstante, la propuesta de reforma educacional demora su fomulación debido a las espec ificida des y ritmos del espa cio político, muy distintos a la polític a de la calle. Inic ia do el gobiemo, se generó un distanciamiento con la oposición política (Alianza por Chile), por el retiro del proyec to de ley del gobiemo anterior, de una Superintendencia de Educación siendo reemplazado por un proyecto para planteles en crisis (Ganora, 4 de mayo 2014; Cooperativa, 5 de mayo 2014). Ante esta situación emergen distintas posiciones, en base a disc ursos técnic os y legisla tivos que esc ondían la disputa ideológica y económic a del actual modelo educativo, así como el rol que debe tener el Estado.

Mientras la discusión sobre la reforma de educación superiory gratuidad se va a plazando, los a c tores polític os y técnic os van redefiniendo en sus polémicas, la a rquitectura conceptual de la misma, pasando de los alcances nomativos de la demanda callejera por una educación pública gratuita de carácter universal a una discusión tecnocrática sobre las posibilida des ec onómic as y polític as de su implementación. En esto, los think ta nks de centro derecha, jugaron un rol fundamental para encauzar la discusión públic a de los distintos proyectos de reforma educacional, hacia un terreno de reforma en los marcosdel modelo económic o y político, disputando la propiedad del problema de la educación y su posible solución. Disolviendo el lazo de responsabilidad causal y política del mundo téc nic o político que la movilización estudiantil había logrado establecer.

Con la discusión política de la reforma, el mundo político fue capaz de desplazaral movimiento estudiantil de la propiedad del problema (Gusfield, 1981; Cefaï, 2011), instituyendo una a rena institucional público-política en la que participan junto al gobiemo, el mundo parlamentario, los partidos políticos de todo el espectro, el movimiento estudiantil, los rectoresuniversita rios, la sorga nizac iones de deud oresy otrosa c tores del sistema educ ac ional. Este desplazamiento se manifiesta en la denuncia explícita que hace el movimiento estudiantil por la nula incidencia en la elaboración del primer proyecto de reforma al lucro, la selección y al copago, que se presentó en mayo 2014. Para el mundo estudiantil, el 
proyecto no atacaba los pilares de la educación de mercado en pos del fortalecimiento de la educación pública (Ulloa, 18 de mayo 2014). Ante dic ha situa ción, el Ministro de Educa ción del periodo, N. Eyzaguirre, ela boró un Plan de Partic ipa ción Ciudada na del Mineduc, para buscar los puntos en común (Moreno, 5 de junio 2014), con la moviliza ción estudia ntil. El mundo universitario, a través de la presidenta de la FECH, Melissa Sepúlveda, expresó sus distanciascon la propuesta al no servinculante, lo que terminó en la salida de la CONFECH del Plan. Para Sepúlveda:

«dejaremos el Plan si es que las garantías no se cumplen, si es que se insiste en tendermanos a la participación y enviar proyectos de ley inconsultos a la vezo si se relega al Plan al rol de un mero club de debate sobre educación» (Sepúlveda, 13 de agosto 2014).

La desconfianza estudiantil hacia el mundo polític o se refuerza por la criminalización de estudiantes, ante la serie de tomasy parosque se inician en junio y julio del año. Desconfianza que alcanzó a la «bancada estudia ntil»que incluía a exdirigentesestudiantiles (G. J ackson, C. Vallejosy K. Ca riola) quienes rec ib ieron fuertes crític as desde la sposic iones más ra dic a les de la movilización, en un contexto en que se organizaba el Congreso por la Educación para los Pueblos, instancia de la que se restaron algunas facciones estudiantiles, como el Bloque de Conducción y la CONES. El actor estudiantil comienza a fragmentarse, perdiendo unidad en las diferentes arenas que el problema se disputa, y con ello, la demanda por el derecho a la educación públic a pierde centralidad.

A fines del 2014 cambian las prioridades del gobiemo en materia de educación, con la disc usión polític a sobre carrera docente, sin algún indic io de la reforma de educación supeniory gratuidad. El gobiemo se propuso el 2015, enviartresproyectos: 1) Nuevo Plan Docente; 2) Desmunic ipalización y; 3) Gratuidad en Educa ción Superior. Con el tercero se sentańan las bases de la futura reforma. No obstante, la alta conflictividad del proyecto de Carrera Docente, tuvo prioridad en la agenda polític a de ese año, por lo que las otras líneas de propuesta en educa ción fueron pospuestas. El conflic to con los doc entes significó la salida del Ministro de Educación Nicolás Eyzaguire, siendo reemplaza do por Adriana Delpiano, quien se hizo cargo del proyec to de reforma de la educa ción superior, limitá nd olo a la idea de gratuidad.

En cuanto a educ a ción superior, se foc a lizó la disc usión polític a y públic a en el financia miento de la propuesta, bajo una idea de gratuidad sin mayor especific ación de su significa do. A partir de lo anterior, el conflic to se desplegó pormedio de discusionestécnicasy parlamentarias, que condic ionaron el diseño de la reforma, enfoca das en las disputas entre rectores de instituc iones públic as y privadas, sobre la redistribución de fondos públicos. El problema de la educación pública como un derecho, definido por los estudiantes, comenzaba su fragmentación.

«Cuando a sume su segundo periodo [Bachelet] genera la sensación de que se apropian de las demandas los movimientos sociales y.... como movimiento estudiantil (y...) se empieza a ver el tema de la educación gratuita, de la gratuidad. Pero en 
cómo se instala. Lo que se genera no es lo que se buscaba (...), se colocó un parche» (Estudiante universita ria, universidad pública, zona sur)

El diseño de reforma a la educ a ción superior del gobiemo sufrió varias modific a ciones en la disc usión política y la a plicación de criterios económicos, especialmente el porcentaje de beneficiarios por la gratuidad. El compromiso de gobiemo fue de un $70 \%$ el 2016 , con un a umento gradual hasta llegar a la universalidad. Sin embargo, posteriomente propone una baja al $60 \%$ y sólo incluye a las universidades del CRUCH. En agosto, la Presidenta a nunc ió que la gra tuidad sería para el $50 \%$, e incluyó a universida despriva da sque cumplieran ciertos requisitos no especific ados. A fines de agosto se convocó al Consejo Consultivo para discutir la Reforma a la Educación Superior (Cooperativa, 27 de agosto 2015), que inc luyó a S. Bitar-ex Ministro de Educa ción del Gobiemo de Rica rdo Lagos-. La participación de Bitar, aumentó la desconfianza y distancia del movimiento estudiantil, porque como dijo la exdingenta V. Sa a vedra a un matutino, «personajescomo Sergio Bitar son los responsables de que miles de compañeros estén marchando, porque están endeudados hasta las patass (La Tercera, 28 de agosto 2015).

La inclusión de los responsables directos del endeudamiento del mundo universitario, fue otra derrota para la movilización estudiantil, que marca completamente la pérdida de propiedad del problema público, junto a la falta de incidencia en la reforma. Finalmente, el 2016, la reforma no tuvo el consenso polític o nec esario y su a plic a ción debió serporglosa presupuestaria, como recurso del ejecutivo para su implementación.

Ese año comienza con un limita do número de benefic ia riosporla gra tuidad, lo que reactivó las protestas estudiantiles. Situa ción que se tra dujo en va rias tomas simbólicas de edificios públicos. En Santiago hubo un enca denamiento en el frontis del Mineduc, en Concepción se tomaron la Seremi de Educación, en Valdivia intervinieron la Secretaráa Regional Ministerial de Educación. Tales acciones de protesta, expresan los esfuerzos de la movilización estudiantil por retomar la propiedad del problema de la educa ción superior (Pa redes, 2019), contra el mund o téc nic o-polític o y otros a c tores (como algunos rectores de planteles privados), tratando de volver a resituarlo en la calle. Sin embargo, la a rena público-política de carácter institucional fue conformada desde la implementación de la gratuidad porglosa. Por ejemplo, los rectores de las diversas c asas de estudios siguen disputando el problema del fina nciamiento de la gratuidad, formulando diferentes concepciones de la educación pública (estatal, pública, de orientación pública). Lo que deja ver la fragmentación de la demanda estudiantil por el derecho a la educación.

La fragmentación de posic iones no solo fue entre los rectores, también oc urió al interior del bloque de gobiemo. En la Comisión de Educación del Senado, la Democracia Cristiana (PDC), perteneciente a la nueva mayoría, entregó su apoyo la educación privada en un escenario legal que no resuelve aún el tema del luc ro, tensionando al bloque ofic ia lista. Lo que demuestra las varia das posic iones que disputan el sentid o del problema educativo. Su posición fue:

«salva guardara la educación como un bien público, pero recalcando que puede ser 
provista de forma estatal y privada. Además, plantean que se respete la libertad de enseñanza, la autonomía universitaria y que se limite lo que califican como una sobremegulación de los planteles de educación superior» (CNN, 9 de mayo 2016).

Con lo anterior, la demanda por una «Educa ción Pública Gratuita», queda dividida en dos problemáticas legislativas diferenciadas, al interior de la arena político-institucional: a) la gratuidad aplicable a instituciones que cumplan ciertos requisitos; b) el de la Educación Pública (Estatal) y su financiamiento. En respuesta, la facción más radical de la movilización estudiantil, respondió con la ofensiva estudiantil (Cooperativa, 24 de mayo 2016), al evaluar como insignific antes las respuestas del gobiemo, implementando medidas de acción directa. Esto tensionó aún más las rela cionesal interior del mundo estudiantil, generando que alguna suniversida des y esc uelas no a dhineron al paro ind efinido convoca do porla CONFECH. La radicalización de las acciones en la ofensiva estudiantil, también generó la pérdida del respa ldo de la ciuda danía ${ }^{7}$. Los estudia ntesperdieron el a poyo del público que a ños a ntes reforza ba sus ma nifestac iones públic as (Pa redes, 2019). Sin embargo, la movilización logró recomponer un atisbo de unidad y fuerza, con las protestas de estudiantes de universida des privadas, a lgo lejanas de los debates más ideológicos de la CONFECH.

El proyecto de ley de educación superior del Gobiemo, no castigó el lucro en educación con cárcel, considerándolo una falta a dministrativa. Tampoco definió el término de la deuda educ a tiva. Ambos puntos centra les para el mundo estudiantil universita rio, lo que a crecienta sus distancia s c on el mund o polític o. Aunque a fines del 2016, se produce la incorporación de la eliminación del CAE en la propuesta, debido a la presión de la movilización estudiantil universitaria de planteles privados, cuyo motivo fue el endeudamiento. Sin embargo, esto no fue suficiente para los movilizados:

[...] en simples palabras lo que se estaba proponiendo por parte de la reforma venía a desvirtuar la demanda estudiantil, o sea generaron en el imagina rio de que la gratuidad ya se había dado, que no siguiéramos reclamando. Pero la gratuidad nunca se dio, o sea para nosotros eso es una falsa gratuidad, que mantiene la lógic a subsidiaria. Nosotros queńa mos cambiar el rol del Estado a un rol garante, que nos garantizara la educación a través de estos aportes basales directos, que nos permitiera como poder sobrevivir de mejor forma en esta lógic a de competencia al interior del mercado». (Estudiante universitario, zona centro, universidad estatal).

El 2017, la moviliza ción estudiantil reconoce su incapacidad para incidir en la formulación de la reforma de educación en una futura discusión política marcada por las elecciones presidencia les del c ic lo posterior. Aunque, las demandas estudiantiles son ca pitaliza das en la consolidación de una fuerza polític a con alcance electoral, el Frente Amplio, que asumió el derecho a la educa ción y la defensa de la educa ción pública como ejesde su programa de gobiemo. Finalmente, en marzo del 2018, la reforma de educación superior fue a probada, en un nuevo gobiemo de derechas sin el apoyo del mundo estudiantil.

\footnotetext{
${ }^{7}$ Ver CADEM N.ํ 127 de la Plaza Pública, que presenta la desaprobación hacia el movimiento estudiantil.
} 


\section{Conclusiones}

A partir del despliegue del problema del derecho a la educa ción universita ria en una arena político-institucional, que excluye a la lógica de la calle en su definición, se pueden derivar a lguna s consecuencias tanto en su configura ción como para la movilización estudiantil, al contrastarlo con el periodo a nterior. En primer lugar, las categoriza ciones de la arena callejera fueron desperfiladas. Por ejemplo, el vocabulario de los derechos fue reconducido al lenguaje técnico-económico, lo que teminó por subsumirlo a la forma neoliberal, y con ello, la causa estudiantil en su lógica de derechos se volvió difusa, según lo expone una de las entrevistadas «no esperábamos mucho, y en verdad es muy triste que con la presión social que había no se hubiese logrado en verdad nada, másque el cambio de nombre de las becasa gratuidad» (Estudiante universita ria, universidad pública, zona centro).

Segundo, la demanda porla educación pública gratuita quedó limitada al tema de la gratuidad, sin que el contenido públic o en ella tuviera relevancia ni especific idad. Lo inespecífic o del debate sobre el estatuto público de las universidades y la indefinición del concepto de lo públic o, teminó en una reforma a las universidades estatales sin mayor impacto e incidencia.

En tercer lugar, el actor colec tivo «estudiantes moviliza dos», con fuerte presencia hasta el 2013, perdió unidad y respaldo público, por ende, sus acciones no tuvieron la resonancia del momento anterior, con ello su incidencia decreció fuertemente, ta mbién su disposición moral y emocional (ver cita más a miba). El público ciudadano, muy activo en el primer ciclo- incluso llego a ser una ciudadanía activista (Paredes, 2018)-, se transformó en una audiencia pasiva de las discusiones de la a rena institucional en el segundo ciclo. Esto se tradujo, para la movilización estudiantil, en la pérdida de propiedad del problema público a manos de otros a ctores, como el gobiemo, los partidos polític os y sus expertos.

Cuarto, los modos de publicitación de la causa pasaron de la manifestación pública a la partic ipa ción en columnas de opinión en medios ma sivos, redes soc ia les y deba tes legislativos e institucionales, donde la forma crítica y nomativa de la discusión fue desplazada por una tecnocrática, que reafimó la categorización neoliberal. Por lo mismo, la conformación de una a rena política-institucional de la gratuidad, al excluir la demanda por el derecho a la educación, limitó las posibilidades de transformación a los marcos institucionales, recuperando en parte, como quinto punto, la lógica de enfrentar los conflic tos previa al 2011, es decir, una resoluc ión elitista que margina a losa c tores socia les de la soluc ión de los conflic tos. Sin embargo, nuestro a nálisis sugiere que, en el a nterior proceso, se ha desarrolla do un desacople entre las respuesta sinstituciona les y la gramática pública, a la luz de las actuaciones de ciertos actores colectivos, que puede tener repercusiones sociopolíticas importantes de cara al futuro.

Para el caso del problema del derecho a la educación superior, consideramos que todo nuevo conflic to relativo a la situación universita ria se relacionará con la reemergencia de la moviliza ción estudiantil y la causa promovida en el ciclo 2011-2017, debido al desacople 
entre gramática públic a orienta da a la lógic a de losderechosy unasrespuestasinstituciona les limitadas por el marco neoliberal. De tal forma, puede que hoy la movilización estudiantil no se encuentre en la primera línea de demandas ciudadanas, pero su causa sigue pendiente y su reconfiguración estará anclada en la lógica de los derechos.

A partir de la periodización propuesta, los efectos de la moviliza ción estudiantil en la gramática pública y el modelo institucional de la educación podrán ponderarse con mayor precisión. De todos modos, el escenario sociopolítico actual en Chile se encuentra abierto a la reactua liza ción del conflic to por la Educación Pública Gratuita, más después del estallido so cial del 18-O. Ello implica contar con un instrumental a decua do para captar el proceso de problematiza ción de causas públicas, las dinámic as del conflic to, las disputas entre actoressocia lesy polític os en la definición legítima del problema o de las solucionesque se planteen. Un instrumental que, a partir de actores sociopolíticos y sus dinámicas relacionales, permita realizar un seguimiento al conflicto en su despliegue coyuntural y procesual, con la finalidad de describirlo e interpreta rlo de manera plausible. Desde nuestra óptica, la perspectiva de los problemas públicos permite realizar tal labor.

De acuerdo a lo argumenta do, leer el conflic to reciente por la educación pública en Chile como un problema público configurado a partir de la actuación y publicitación de los propios estudiantes movilizados, permite un mayor rendimiento comprensivo de los procesos socio polític os para ponderar las formas públicasy polític as que son desplega daspor los ac tores partícipes del c onflic to, al tiempo que permite observar sus log ros y limitac iones en a renas públic as delimitadas. Es una herramienta de utilidad, porque define temas, actores, espacios, relacionesy efec tosen situa ciones de controversia sy puede potenciar nuestras interpretaciones de los conflic tos soc iopolític os, a partir de su imbricación e intersección mutua.

Consideramos que estudiar el conflicto social a partir de las trayectorias de problemas públic os espec ífic os desplega dos en a renas definidas, pero que en su conjunto cuestionen las bases neoliberales de la democracia, pueden contribuir a interpretar mejor y explicar con más detalle el reciente contexto de revueltas sociopolític as en Chile.

\section{Agradecimientos}

Este a rtíc ulo ha sido posible gracias al financiamiento de ANID en Chile, mediante el proyec to FONDEC YTPostdoc tora do 3170504.

\section{Bibliografía}

Aguilera, O. (2011). Acontec imiento y acción colectiva juvenil. El antes, durante y después de la rebelión de los estudiantes secundarios chilenos en el 2006. Propuesta Educativa, 1, (35), 11-26.

Ala rc ón-Leiva, J., J ohnston, E. y Frites-C a milla, C. (2014), Consenso polític o y pac to 
educ ativo. Pospolític a y educa ción en Chile (1990-2012). Universium, 29, (2), 37-48. Recuperado de http://dx.doi.org/10.4067/S0718-23762014000200004.

Alexander, J . (2010). Ic onic Consciousness: The material feeling of meaning. Thesis Eleven, 103(1), 10-25.

Ara ujo, K., y Martuccelli, D. (2012). Desafíos comunes. Retrato de la sociedad chilena y sus ind ividuos. Sa ntiago, Chile: LOM.

Assaél, J .; R. Comejo; J. González; J. Redondo; R. Sánchez \& M. Sobarzo (2011). La empresa educativa chilena. Educação \& Sociedade, 32(115), 305322

Atria, F. (2013). El neoliberalismo con rostro humano. Santiago, Chile: Catalonia. (2014). Derechos sociales y educación: el nuevo paradigma de lo públiCo. Santiago; Chile: LOM.

Báez Urbina, F. (2017). Diseño institucional y neoliberalismo. El modelo chileno como resultado del quiebre unilateral del contrato social. Revista de Sociologia, 102, (3), 449-476, Recuperado de http://dx.doi.org/10.5565/rev/ papers.2299

Braghetto, M. (2013). El movimiento universitario chileno y las transformaciones de la educación superior en el Chile neoliberal. Revista Izquierdas, 16, 55-74.

C efaï, D. (2011). Diez propuestas pa ra el estudio de las moviliza ciones colectivas. Contexto de experiencias y problemas públicos. Revista de Sociología de la Universidad de Chile, (26), 137-166. Recuperado de DOI: 10.5354/0719529X.2011.27491

(2014). Investigar los problemas públicos con y más allá de Joseph Gusfield. En J. Gusfield, La cultura de los problemas públicos, el mito del conductor alcoholiza do versus la sociedad inocente. Buenos Aires, Argentina: Siglo XXI.

Donoso, S. (2014). La reconstruc ción de la acción colectiva en el Chile post transic ión: el caso del movimiento estudiantil. CLACSO.

Favre, P. (1990). La manifestation. Paris: Presses de la Fondation Nationale des Sciencias Politiques.

Figueroa, F. (2013). Legamos para quedamos. Crónica de la revuelta estudiantil. Santia go, Chile: LOM.

Garfinkel, H. (2006). Estudios de etnometodología. Madrid, España: Anthropos.

Garretón, M., y Espinosa, M. (1992). ¿Reforma del Estado o cambio en la matriz socio-política?. Sa ntiago, Chile: FLACSO. Recuperado de: http:// fla c so c hile.org/bibliotec a/pub/memoria/1992/000480.pdf

Gusfield, J. (1981). The culture of public problems: the drinking driving problem and the production of simbolic order. Chicago, USA: University of Chic ago Press.

Hilgartner, S., y Bosk, C. (1988). The rise and fall of sociol problems: a public arenas model. The American J oumal of Sociology (94), 53-79.

Isin, E. (2009). Citizenship in flux: The figure of the a ctivist c itizen. Subjectivity, 29(1), 367-388.

Jackson, G. (2013). El país que soñamos. Santiago, Chile: Debates.

Jaspers, J. (1997). The a rt of moral protest: cultura, biography, and creativity in social movements. Chicago, USA: University of Chicago Press.

Krememan, M., \& Páez, A. (2016). Endeudar para gobemar y mercantilizar. El caso del CAE. Santiago, Chile: Fundación Sol.

Lechner, N. (2003). Esta do y sociedad en una perspectiva democrática. Polis. Revista La tinoamericana, (6), . DOI: http://dx.doi.org/10.32735/S0718-6568/ 2003-N6-246

Lemieux, R. (2017). Gramáticas de la acción social. Buenos Aires, Argentina: Sig lo $X X I$.

Madariaga, A. (2019). La continuidad del neoliberalismo en Chile: ideas, instituciones e intereses. Revista de Estudos e Pesquisas sobre as Américas. 13 (2), 81-113. Recuperado de https://doi.org/10.21057/10.21057/repa mv $13 n 2.2019 .23217$

Montero, V. (2018). Movimientos so c ia les y c onsec uenc ias polític o instituc iona les. Del movimiento universitario 2011 a la Reforma en educación superior en Chile. Persona y Sociedad, 32 (2), 46-88.

Moulián, T. (1997). Chile a ctual: a natomía de un mito. Santiago, Chile: LOM.

Muñoz, V., \& Durán, C. (2019). Los jóvenes y los movimientos estudia ntiles en el 
Chile reciente. Cic los soc iopolític os entre 1967 y 2017. Revista Izquierdas, (45) 129-159.

Nardacc hioni, G., \& Acevedo, M. (2013). Las sociologías pragmático-pragmatistas puestas a prueba en América Latina. Revista Argentina de Sociología (18), 87-118.

Narda cc hioni, G y y Márquez, A. (2016). La c a lle y el Parlamento: senderosque se unen y bifurcan. El conflic to educativo en Argentina y el problema del aborto en Méxic 0. Terc eiro Milênio: Revista Crític a de Sociologia e Política, 6, (1), 157-197.

Paredes, J.P. y Otárola, C. (2019). El grito ciudadano en la lucha por el derecho a la educación: El marco moral y emocional de la movilización estudiantil en Chile (20112013). Sociológica, 98, (34), 255-291.

Pa redes, J.P. (2011). Ciuda da nía, partic ipación y democracia. Deuda y déficit en los 20 años de «democ ra cia »en Chile. Revista Polis, 28(10), 474-499. DOI: http://dx.doi.org/ 10.32735/S0718-6568/2011-N28-773

Paredes, J.P. (2013). ¡M oviliza rse tiene sentido! Análisis cultural en el estudio de las movilizaciones sociales». Psic operspectivas, 12, (2), 16-27. Recuperado de DOI: 10.5027/PSIC OPERSPEC TIVAS-VOL12-ISSUE2-FULITEXT-279

Paredes, J.P. (2018). En la calle y sin permiso, yo me educo y organizo. La manifestación por la Educación Pública como forma de politización de la juventud chilena. en R. Torres (ed.) Juventud, espacios públic os y partic ipación en Chile y América Latina. Santiago de Chile. Santiago, Chile: UCEN/RIL Editores.

Paredes, J .P, Ortiz, N. y Araya, C. (2018). Conflicto social y subjetiva ción política: performance, militancias y memoria en la moviliza ción estudiantil post 2011. Persona y Sociedad, 32, (2), 122-149.

Picazo, M. I., \& Pierre, C. (2016). La educa ción como derecho social: la construcción del referencial de acción pública del movimiento estudiantil chileno. Revista Uruguaya de Ciencia Política, 25(2), 99-210.

Ramírez, S. (2017). Los derechos sociales como crítica del modelo neoliberal: anta gonismos emergentes en el Chile Actual. Revista Academia y Crítica, 1(1). Recuperado de http://dx.doi.org/10.25074/07199147.1.572

Rojas, J. (2012), Sociedad bloquea da. Movimiento estudiantil, desigualdad y despertar de la sociedad chilena. Santiago, Chile: RIL eds.

Rovira, C. (2007). Chile: transic ión pacta da y débil autodetermina ción colectiva de la sociedad. Revista Mexic ana de Sociología, 69(2), 343-372.

Sc hilla gi, C. (2011). Problemas públic os, casos resonantes y escándalos. Algunos elementos para una discusión teónica. Polis, 30 (10), 245-266. Recuperado de http://dx.doi.org/10.4067/S0718-65682011000300012

Segovia, C., y Gamboa, R. (2012). Chile el año que salimos a la calle. Revista de Ciencia Política, 32(1), 65-68.

Slachevsky, N. (2015), Una revolución neoliberal: la política educacional en Chile desde la dictadura militar. Educação e Pesquisa, 42, (spe), 1473-1486. Recuperado de http://dx.doi.org/10.1590/S1517-9702201508141660

Spector, M., y Kitsuse, J . (1977). Construc ting so cial problems. New York, USA: Aldine. Thielemann, L. (2017). La izquierda radical y el movimiento estudiantil chileno de fin de siglo: tra nsforma ciones, orga niza c ión y reflexiones crític as. En R. Ma rsike, Movimientos estudiantiles de América Latina V, pp. 115-154. Ciudad México, Méxic o: IISUE - UNAM

Tilly, C. (2008). Contentious performances. Cambridge, Ingla terra: Cambridge University Press.

Trom, D. (2008). Gramática de la movilización y vocabularios de motivos. En A. Natalucci, La comunic ación como riesgo. La Plata, Argentina: Al Margen.

Vera, S. (2011). Nuevos Movimientos Sociales y Combinación de paradigmas polític os en democracias postd ic ta toriales: El caso del movimiento estudia ntil en Chile 2006. Conflic to Social, 4 (5), 374-406

\section{Prensa}

CNN. (9 de mayo, 2016). Democracia Cristiana entrega propuestas para educación superior. CNN. Recuperado de https://www.cnnchile.com/pais/democracia-c ristia na-entrega-pro puesta s-para-educa cion-superior_20160509/

Cooperativa. (5 de mayo, 2014). Presidenta fimó proyecto que creā el interven- 
tor educa cional para planteles en crisis. Cooperativa. Recuperado de https:/ / www.coopera tiva.cl/noticia s/pa is/ educa cion/presidenta-fimo-proyectoque-crea-el-intervento r-educac ional-para/2014-05-05/094003.html

(27 de agosto, 2015). Mineduc convocó «Consejo Consultivo para la Reforma en Educación Superions. Cooperativa. Recuperado de https:// www.cooperativa.cl/noticias/pa is/educacion/proyectos/mineduc-convococonsejo-c onsultivo-pa ra-la-refo ma -en-ed uc a c ion/2015-08-27/ 154816.html (24 de mayo, 2016). Estudia ntes sec unda rios y universita rios imumpieron en el Palacio de La Moneda. Cooperativa. Recuperado de https:// www.coopera tiva .cl/notic ias/pa is/educa c ion/movimiento-estud ia ntil/estudiantes-sec unda rios-y-universita rios-imumpieron-en-el-pa la c io-de-la/2016-0524/095635.html

Ganora, E. (4 de mayo, 2014). Ejecutivo despliega ofensiva en el Congreso para defender reformas clave. La Tercera. Recuperado de https:// www.la tercera.com/noticia/ejec utivo-despliega-ofensiva-en-el-cong resopara-defender-refomas-clave/

La Tercera. (28 de agosto, 2015). Confech: personajes como Sergio Bitar son los responsables de que miles de compañeros estén marchando. La Tercera. Rec uperado de https:// www.latercera.com/noticia/confech-personajescomo-serg io-b ita r-son-los-resp onsa bles-de-que-miles-de-compa neros-estenmarchando/

Mańn, V. \& Ramírez, N. (18 de julio, 2017). Delpiano ante críticas de Jackson y Boric al proyecto de educación superior: «Aquí no hay ninguna trampa». Emol. Recupera do de https://www.emol.com/notic ias/Nacional/2017/07/18/ 867350/Delpia no-a nte-critic a s-a I-proyec to-de-educ a c io n-superior-Aqui-nohay-ning una -trampa.html

Moreno, G. (5 de junio, 2014). Eyzaguirre lanza propuesta de participación y convoca a actores de la educación para incidir en la reforma. La Tercera. Recuperado de https://www.latercera.com/noticia/eyza guirre-la nza-propuesta-de-partic ipa cion-y-convoca-a-actores-de-la-educa cion-pa ra-incidir-en-la-reforma/

(4 de agosto, 2014). Revolución Democrática y J uventudes Comunistas conmemoran hoy el «Día de la Dignidad Estudiantil». La Tercera. Recuperado de https:// www.latercera.com/noticia/revolucion-democratica-yjuventudes-comunista s-c onmemo ran-hoy-el-dia-de-la-dignida d-estud ia ntil/

Sepúlveda, M. (13 de a gosto, 2014). No queremos regular, queremos cambiar el modelo. Diario Universidad de Chile. Recuperado de https://radio.uchile.cl/ 2014/08/13/no-queremos-regula r-queremos-ca mbiar-el-mo delo/

Ulloa, G. (18 de mayo, 2014). Presidenta de la FECh: «Hoy día tiene que existir política real»pa ra revertir la c risiseduc a cional. Bio-Bio. Rec upera do de https:/ /www.biobio chile.cl/notic ia s/2014/05/ 18/presidenta-de-la-fech-hoy-dia-tiene-que-existir-po litic a -rea l-pa ra-revertir-la -c risis-e duc a c io na I.shtml 